

\section{Critical material attributes (CMAs) of strip films loaded with poorly water- soluble drug nanoparticles: I. Impact of plasticizer on film properties and dissolution}

Scott M. Krull, Hardik V. Patel, Meng Li, Ecevit Bilgili, Rajesh N. Davé*

Otto H. York Department of Chemical, Biological, and Pharmaceutical Engineering, New Jersey Institute of Technology, Newark, NJ 07102, USA

$15 *$ Corresponding author. Tel.: +1 -973-596-5860; fax: +1 -973-642-7088.

E-mail address: dave@njit.edu (Rajesh N. Davé). 


\section{Introduction}

Polymer strip films have become an increasingly popular method of drug delivery in

20 recent years. In addition to offering a larger surface area for faster disintegration and dissolution (Dixit and Puthli, 2009), films also offer several formulation, manufacturing, and consumerbased advantages over more traditional solid dosage forms. Noteworthy manufacturing advantages include inherently continuous processing and flexible, cost-effective scale-up (Hoffmann et al., 2011) while consumers, particularly pediatric, geriatric, and dysphagic

25 patients, have demonstrated a higher degree of compliance for film-based pharmaceuticals (Dixit and Puthli, 2009). One of the most intriguing aspects of the strip film format, however, resides in its versatility. Strip films benefit from access to a wide variety of usable excipients, including film-formers and plasticizers, which can be used to control various properties of the films without sacrificing the integrity of the format (Borges et al., 2015b; Dixit and Puthli, 2009).

30 However, little is known about how these critical material attributes (CMAs) impact properties of drug particle-laden films, such as drug particle stability and dissolution rate.

Generally speaking, plasticizers are additives that increase the plasticity or fluidity of a material. In strip films, this is typically achieved by introducing a plasticizer in the form of a relatively small molecule that is miscible with the film-forming polymer, allowing for molecular-

35 level interaction between the plasticizer and polymer to moderate polymer-polymer interaction, thereby promoting mobility of the polymer chains (Bruce and McGinity, 2008). This is manifested in a depression of the glass transition temperature, as well as decreased tensile strength and increased elongation in the resulting films (Wypych, 2004). This phenomenon has been reported for a multitude of polymer-plasticizer combinations, but such studies have mainly

40 been limited to drug-free films (Aulton et al., 1981; Bodmeier and Paeratakul, 1994; Entwistle 
and Rowe, 1979; Gutiérrez-Rocca and McGinity, 1994; Honary and Orafai, 2002; Hutchings et al., 1994; Hyppölä et al., 1996; Lim and Hoag, 2013; Lourdin et al., 1997; McHugh and Krochta, 1994; Sakellariou et al., 1986; Thakhiew et al., 2010). Plasticizers are particularly useful in pharmaceutical films, as most film-forming polymers produce hard or brittle films in the absence

45 of plasticizer, resulting in handling/packaging difficulties and poor patient compliance. This has led to investigations of the impact of plasticizer on various properties of polymer films loaded with water-soluble drug (Gottnek et al., 2013; Pongjanyakul and Puttipipatkhachorn, 2007) and amorphous poorly water-soluble drug (Panda et al., 2014). However, this effect has yet to be investigated for films containing poorly water-soluble drug particles, let alone the effect of 50 plasticizer or plasticizer content on drug release from such films.

While most literature and commercial applications involving polymer films for drug delivery have focused on orodispersible films for water-soluble drugs (Borges et al., 2015a; Dave et al., 2014; Dixit and Puthli, 2009; Garsuch and Breitkreutz, 2010), the potential for successful delivery of poorly water-soluble drugs via strip films had gone untapped until

55 recently. Use of poorly water-soluble drugs introduces two additional challenges to drug delivery: overcoming limited solubility to improve bioavailability (Kesisoglou et al., 2007) and ensuring that the enhanced bioavailability is preserved upon delivery (de Villiers, 1996; Krull et al., 2015a). The two most commonly used methods to overcome these challenges, organic solvent casting (Kumar et al., 2014; Visser et al., 2015) and hot melt extrusion (Prodduturi et al., 60 2005), both pose inherent limits to drug loading in the film and may lead to instability of the embedded drug (Kipp, 2004). Several particle engineering techniques have demonstrated promise in terms of producing stable poorly water-soluble drug particles for incorporation into and fast dissolution from polymer strip films with less stringent limitations on drug loading, 
including production of drug nanoparticles via wet stirred media milling (WSMM) (Krull et al.,

65 2016; Krull et al., 2015b; Sievens-Figueroa et al., 2012a; Susarla et al., 2015; Susarla et al., 2013), high pressure homogenization (Lai et al., 2015; Shen et al., 2013), liquid antisolvent precipitation (Beck et al., 2013), and melt emulsification (Bhakay et al., 2016). However, none of these studies investigated the impact of plasticizer on film properties as well as dissolution rate.

The objective of this work is to demonstrate that the mechanical properties of polymer strip films loaded with poorly water-soluble drug can be manipulated within a desirable range without sacrificing fast dissolution or nanoparticle redispersibility of the embedded poorly watersoluble drug. Aqueous nanosuspensions of griseofulvin, used as a model poorly water-soluble drug, were prepared via WSMM. Griseofulvin nanosuspension was then mixed with

75 hydroxypropyl methylcellulose polymer solution containing varying amounts of glycerin, triacetin, and polyethylene glycol as plasticizers. The resulting film precursor suspensions were cast and dried to form films loaded with griseofulvin nanoparticles. The viscosity of each film precursor suspension was measured using a coaxial cylinder rheometer. The size distributions of griseofulvin particles redispersed from films into deionized water were compared to that of the milled griseofulvin nanosuspension, all measured via laser diffraction, to assess the ability of the film format to physically stabilize the griseofulvin nanoparticles. Differential scanning calorimetry was used to determine the glass transition temperature of the films. Mechanical properties, including tensile strength and percent elongation at break, were also measured for all films. Film content uniformity was assessed via assay. Film dissolution rate was measured using 85 a USP-IV flow-through cell dissolution apparatus. Thermogravimetric analysis was employed to 
analyze residual moisture content of the films. Drug particle size and morphology was investigated qualitatively using scanning electron microscopy.

\section{Materials and methods}

$90 \quad 2.1 \quad$ Materials

Griseofulvin (Letco Medical, Decatur, AL, USA) was selected as a model BCS class II drug. Hydroxypropyl methylcellulose (HPMC; Methocel E15 Premium LV, The Dow Chemical Company, Midland, MI, USA) served as the film-forming polymer. HPMC-E15LV also served as a nanoparticle stabilizer in suspension, along with the surfactant sodium dodecyl sulfate (SDS;

95 Fisher Scientific, Pittsburgh, PA, USA). Glycerin (Sigma-Aldrich, St. Louis, MO, USA), triacetin (Sigma-Aldrich, St. Louis, MO, USA), and polyethylene glycol (PEG-400; SigmaAldrich, St. Louis, MO, USA) were selected as film plasticizers for their miscibility with HPMC and limited volatility. Particle size reduction of griseofulvin was performed by WSMM according to Section 2.2.1. All other materials were used without further processing.

100

\subsection{Preparation methods}

\subsubsection{Preparation of griseofulvin nanosuspension}

Griseofulvin nanosuspension was prepared via WSMM using a Netzsch mill (Microcer, Fine particle technology LLC, Exton, PA, USA). Methods and stabilizer concentrations were 105 selected according to previous optimization studies (Bilgili and Afolabi, 2012; Monteiro et al., 2013). The suspension consisted of $10 \%$ griseofulvin (w/w wrt water) dispersed in a stabilizer solution of 2.5\% HPMC-E15LV and 0.5\% SDS (both w/w wrt water), and was milled for 120 $\min$. 


\subsubsection{Preparation of film precursor suspensions}

Formulations for film-forming polymer solutions are listed in Table 1. As per Dow ${ }^{\circledR}$ protocol, polymer solutions were prepared by adding the appropriate amounts of HPMC-E15LV and plasticizer (as necessary) to water at $90{ }^{\circ} \mathrm{C}$, after which the solution was allowed to cool to room temperature under continuous magnetic stirring. Polymer concentrations were selected

115 such that the polymer solutions were sufficiently viscous to ensure a uniform film (Susarla et al., 2013) while not too viscous to hinder mixing or casting. Polymer solutions consisted of $15 \mathrm{wt} \%$ HPMC with different plasticizers (glycerin, triacetin, and PEG-400) and plasticizer content $(0.0$, 2.5, and $5.0 \mathrm{wt} \%$ ). Plasticizer concentrations were kept at or below $5.0 \mathrm{wt} \%$ in order to avoid over-plasticization of the films, which can result in oily films that are difficult to handle. Each of

120 the resulting polymer solutions was mixed with griseofulvin nanosuspension in a 2:1 ratio by mass using a Thinky ARE-310 planetary centrifugal mixer (Thinky, Laguna Hills, CA, USA). Polymer solution and nanosuspension were mixed at $2000 \mathrm{rpm}$ for $30 \mathrm{~s}$, followed by $7 \mathrm{~min}$ of deaeration at $2200 \mathrm{rpm}$, to form film precursor suspension. If bubbles were still present in the precursor suspension after mixing, the precursor was left overnight to settle before casting.

\subsubsection{Preparation of polymer films containing griseofulvin nanoparticles}

9-10 g of film precursor suspension was manually cast onto a stainless steel substrate with a doctor blade (Elcometer, MI, USA) at a fixed $1000 \mu \mathrm{m}$ thickness. Wet films were then dried in the convective zone of a Lab-Cast Model TC-71LC Tape Caster (HED International, NJ, USA) in batch mode at $50{ }^{\circ} \mathrm{C}$ under laminar air flow for a period of $1 \mathrm{~h}$ (Davé et al., 2014). The 
films were peeled from the substrate after drying and stored in individual sealed plastic bags for characterization.

\subsection{Characterization methods}

$135 \quad 2.3 .1$ Viscosity

The apparent shear viscosity of polymer solutions and film precursor suspensions was measured with an R/S-CC+ Coaxial Cylinder Rheometer (Brookfield Engineering, Middleboro, MA, USA) equipped with a shear rate controlled coaxial cylinder (CC25) and Lauda Eco water jacket assembly (Lauda-Brinkmann LP, Delran, NJ, USA) for temperature control. Both were

140 subjected to a low shear rate program $\left(0-20 \mathrm{~s}^{-1}\right)$ at $25 \pm 0.5^{\circ} \mathrm{C}$ to measure low shear viscosity. Raw data was analyzed using Rheo 3000 software (Brookfield Engineering, Middleboro MA, USA). Experiments were performed a minimum of seven times.

\subsubsection{Griseofulvin particle size after milling and redispersed from films}

Griseofulvin particle size distributions were measured both in suspension immediately after milling and following redispersion from films utilizing a Coulter LS 13320 Laser Diffraction Particle Size Analyzer (Beckman Coulter, Miami, FL, USA) employing a polarized intensity differential scattering (PIDS) obscuration water optical model. The PIDS was maintained between $40-50 \%$ and obscuration was maintained below $8 \%$. Mie scattering theory

150 was used to calculate particle size distributions. Suspension samples for particle size after milling were prepared by removing a $1.2 \mathrm{ml}$ sample from the holding tank of the mill, dispersing the sample into $4 \mathrm{ml}$ of stabilizer solution (2.5\% HPMC-E15LV and $0.5 \%$ SDS, both w/w wrt water) via pipette, and vortex mixing for $1 \mathrm{~min}$ at $1500 \mathrm{rpm}$. 
In order to evaluate the ability to recover griseofulvin nanoparticles from films upon

155 delivery, redispersion samples to determine griseofulvin particle size after incorporation into dry films were prepared by dispersing three circular film punches $\sim 0.7 \mathrm{~cm}^{2}$ in area into $3 \mathrm{ml}$ of deionized water, followed by $3-5 \mathrm{~min}$ of vortex mixing at $1500 \mathrm{rpm}$. These samples were then subjected to the same particle size analysis as the milled suspension samples.

\subsubsection{Differential scanning calorimetry (DSC)}

A Polymer DSC (Mettler Toledo, Columbus, OH, USA) was used for differential scanning calorimetry. 5-6 mg samples were placed in sealed aluminum pans, initially cooled to $50{ }^{\circ} \mathrm{C}$ at a rate of $-10{ }^{\circ} \mathrm{C} / \mathrm{min}$, heated to $250{ }^{\circ} \mathrm{C}$ at $10^{\circ} \mathrm{C} / \mathrm{min}$, cooled to $-50{ }^{\circ} \mathrm{C}$ at $-10{ }^{\circ} \mathrm{C} / \mathrm{min}$, and again heated to $250^{\circ} \mathrm{C}$ at $10^{\circ} \mathrm{C} / \mathrm{min}$, all under nitrogen flow.

165

\subsubsection{Mechanical properties of the films}

Film mechanical properties were measured using a TA-XT Plus Texture Analyzer (Stable Microsystems, UK). Rectangular film strips with dimensions of $50 \mathrm{~mm} \times 15 \mathrm{~mm}$ were held between two grips and stretched at a constant speed of $1 \mathrm{~mm} / \mathrm{s}$ until the point of tensile failure.

170 Tensile strength (TS), yield strength (YS), Young's modulus (YM), and percent elongation at break (EB) were computed from the resulting stress vs. strain data. Data represents an average of four strips.

\subsubsection{Determination of drug content and uniformity in films}

175 Ten circular samples $\sim 0.7 \mathrm{~cm}^{2}$ in area were removed from random points throughout each film and dissolved in $250 \mathrm{ml}$ of $5.4 \mathrm{mg} / \mathrm{ml}$ SDS solution under continuous stirring for a 
minimum of $3 \mathrm{~h}$. The UV absorbance of each sample at the wavelength of maximum absorbance for griseofulvin $(291 \mathrm{~nm})$ was then measured using a Thermo Scientific Evolution 300 UV-Vis spectrophotometer (Thermo Fisher Scientific Inc., MA, USA). The concentrations of the 180 respective samples were calculated via calibration curve. Content uniformity results are expressed as average griseofulvin weight per unit area of film and average weight percentage of griseofulvin in the film over ten samples.

\subsubsection{Dissolution}

Dissolution experiments were performed using a flow-through cell dissolution apparatus (USP IV; Sotax, Switzerland) with cells of $22.6 \mathrm{~mm}$ internal diameter and $0.2 \mu \mathrm{m}$ Pall HT Tuffryn filters. Dissolution samples were automatically measured in-line every two minutes by the same Thermo Scientific Evolution 300 UV-Vis spectrophotometer (Thermo Fisher Scientific Inc., MA, USA) used for determination of drug content and uniformity in films (Section 2.3.5).

190 One circular film sample $\sim 0.7 \mathrm{~cm}^{2}$ in area was horizontally secured within $5 \mathrm{~g}$ of $1 \mathrm{~mm}$ glass beads at the bottom of each cell ( $3 \mathrm{~g}$ below the film and $2 \mathrm{~g}$ above). $100 \mathrm{ml}$ of $5.4 \mathrm{mg} / \mathrm{ml} \mathrm{SDS}$ solution (recommended for griseofulvin by the United States Pharmacopeia) was circulated through each cell at a flow rate of $16 \mathrm{ml} / \mathrm{min}$ and a constant temperature of $37 \pm 0.5^{\circ} \mathrm{C}$. These dissolution protocols were selected for their discriminatory power demonstrated in previous

195 work between films containing nanoparticles, films containing microparticles, and compacted powders (Beck et al., 2013; Bhakay et al., 2016; Sievens-Figueroa et al., 2012b). Dissolution results are reported as griseofulvin release as a function of time for an average of six samples from each film. Percentage griseofulvin released was calculated based on the expected drug 
loading in each sample from the drug content assessment performed for each film formulation

200 (Section 2.3.5), taking into consideration the weight of each individual film sample.

\subsubsection{Thermogravimetric analysis (TGA)}

TGA was performed using a TGA/DSC1/SF STAR $^{\mathrm{e}}$ system (Mettler Toledo, Inc., Columbus, OH, USA). A $\sim 4 \mathrm{mg}$ film sample was placed in a ceramic crucible and heated under

205 nitrogen flow from $25{ }^{\circ} \mathrm{C}$ to $150{ }^{\circ} \mathrm{C}$ at a constant rate of $10{ }^{\circ} \mathrm{C} / \mathrm{min}$, held at $150{ }^{\circ} \mathrm{C}$ for $15 \mathrm{~min}$, heated to $250^{\circ} \mathrm{C}$ at a rate of $10^{\circ} \mathrm{C} / \mathrm{min}$, and finally cooled back to $25^{\circ} \mathrm{C}$ at a rate of $-10{ }^{\circ} \mathrm{C} / \mathrm{min}$.

\subsubsection{Scanning electron microscopy (SEM)}

A field emission scanning electron microscope (FESEM) LEO1530VP GEMINI (Carl

210 Zeiss, Inc., Peabody, MA, USA) was used to observe the potential presence of drug aggregates within the films. A small film sample was mounted to an aluminum stub with carbon tape and carbon coated using a sputter coater (Bal-Tec MED 020 HR, Leica Microsystems, Wetzlar, Germany) prior to imaging the cross section of each film.

\section{$215 \quad$ 2.3.9 Long-term stability of films}

Films designated for long-term stability study were stored in a MicroClimate benchtop climate chamber (Cincinnati Sub-Zero, Cincinnati, OH, USA) at $40{ }^{\circ} \mathrm{C}$ and $75 \%$ relative humidity (RH). Following 3 and 6 months of storage under these conditions, films were subjected to redispersion and dissolution tests as outlined in Sections 2.3.2 and 2.3.6, 220 respectively. 


\subsubsection{Statistical analysis}

All calculations were performed using Microsoft Excel ${ }^{\circledR}$ (Microsoft Office 2010, USA). Results for viscosity, mechanical properties, and dissolution profiles are expressed as mean \pm

225 S.D. (standard deviation) while content uniformity results are expressed as mean with R.S.D. \% (relative standard deviation). Dissolution profiles were compared using similarity and difference factors (Boateng et al., 2012; Costa and Lobo, 2001).

\section{Results and discussion}

\section{$230 \quad 3.1 \quad$ Viscosity of polymer solutions and precursor suspensions}

Low shear $\left(2.2 \mathrm{~s}^{-1}\right)$ viscosity measurements for polymer solutions and film precursor suspensions, performed with the objective of mimicking conditions of film casting, are presented in Fig. 1. The viscosities of the polymer solutions ranged from 9500-14000 cP. Upon dilution with aqueous griseofulvin nanosuspension that contained about $88.5 \mathrm{wt} \%$ water, the viscosities

235 of the film precursor suspensions ranged from 5000-7000 cP. Polymer solutions with 5.0\% plasticizer exhibited greater viscosities than those with $2.5 \%$ plasticizer for all three plasticizers, which may be explained by the fact that the added plasticizers were of greater viscosity $(1400 \mathrm{cP}$ for glycerin, $23 \mathrm{cP}$ for triacetin, and $60 \mathrm{cP}$ for PEG-400) than the water they displaced from the formulation (Table 1). These trends were also observed in the film precursor suspensions, 240 although not as prominently due to dilution with griseofulvin nanosuspension. However, there was a clear decline in polymer solution viscosity upon inclusion of glycerin and triacetin relative to the plasticizer-free polymer solution. This may be the result of easier slippage of polymer chains under shearing due to the initial incorporation of plasticizer, which effectively reduced the extent of polymer-polymer interaction and, consequently, polymer solution viscosity. 


\subsection{Drug particle size after milling and redispersed from films}

Since the purpose of nanomilling poorly water-soluble drug particles is to enhance their dissolution rate and bioavailability, it is crucial that the drug nanoparticles embedded in the polymer matrix do not aggregate prior to or upon delivery. Redispersion testing provides a 250 means by which the size of the poorly water-soluble drug particles released upon delivery can be assessed. As has been shown previously for solid dosage forms containing poorly water-soluble drug nanoparticles (Bhakay et al., 2014; Bhakay et al., 2013; Krull et al., 2016), redispersion offers predictive insight into dissolution, as drug nanoparticle aggregation or growth can lead to slower drug release. In order to investigate the effect of different plasticizers on the ability of the

255 film format to physically stabilize the griseofulvin particles, redispersion tests were performed on all films on the day of preparation, as well as after 3 and 6 months of storage under stress conditions $\left(40^{\circ} \mathrm{C}, 75 \% \mathrm{RH}\right)$ to assess long-term stability (stability results discussed in Section 3.3.7). Particle size statistics for the milled griseofulvin nanosuspension and griseofulvin particles redispersed from films in deionized water after 0,3 , and 6 months of storage are given 260 in Table 2 as 10\%, 50\% (median), and 90\% passing size ( $d_{10}, d_{50}$, and $d_{90}$, respectively).

Griseofulvin particle redispersion from fresh glycerin and PEG-400 films yielded $d_{10}$ values less than that of the milled suspension and $d_{50}$ values between $160-170 \mathrm{~nm}$, on par with that of the milled suspension, both indicating very good physical stability in the film. While the $d_{90}$ values of the same films were between 300-350 nm compared to $262 \mathrm{~nm}$ in the suspension,

265 such differences were expected, as the $d_{90}$ is significantly more sensitive to aggregation. On the other hand, slightly larger nanoparticles were redispersed from fresh triacetin films ( $d_{50}$ of 241 $\mathrm{nm}$ and $321 \mathrm{~nm}$ for $2.5 \%$ and $5.0 \%$ triacetin, respectively) and the plasticizer-free film $\left(d_{50}\right.$ of 
$231 \mathrm{~nm}$ ) compared to those from glycerin and PEG-400 films ( $d_{50}$ between 160-170 $\mathrm{nm}$ ), which increased in size with increasing triacetin content. That said, even in the 5.0\% PEG film, the $d_{90}$

270 value was only $556 \mathrm{~nm}$, and $100 \%$ of all particles were $<1 \mu \mathrm{m}$ in size (full size distribution not shown). As such, these differences in nanoparticle size are not expected to negatively affect drug dissolution rate from films under sink conditions (Krull et al., 2016; Murdande et al., 2015).

\subsection{Film characterization}

\subsubsection{Differential scanning calorimetry (DSC)}

One of the defining features of a plasticizer is its ability to depress the glass transition temperature $\left(\mathrm{T}_{\mathrm{g}}\right)$ of a polymer due to their molecular-level interaction (Bruce and McGinity, 2008; Wypych, 2004). To this end, DSC was employed to observe the influence of plasticizer and plasticizer concentration on the $T_{g}$ of HPMC films loaded with poorly water-soluble drug 280 nanoparticles. However, the DSC signal in HPMC films is very weak (Gómez-Carracedo et al., 2003; Kararli et al., 1990), often making it difficult to observe the glass transition. As such, the first heating cycle was used to determine the $\mathrm{T}_{\mathrm{g}}$ for each film.

The impact of plasticizer and plasticizer concentration on the $T_{g}$ of griseofulvin nanoparticle-laden films can be seen in Fig. 2 (full DSC traces can be found in Supplementary

285 Material). These $T_{\mathrm{g}}$ values are in line with those observed in literature, which range between 150-200 ${ }^{\circ} \mathrm{C}$ depending on the grade of HPMC and method of characterization (GómezCarracedo et al., 2003; McPhillips et al., 1999; Nyamweya and Hoag, 2000). Increasing concentration of all three plasticizers led to a clear reduction in $T_{g}$, a trend which has been observed for multiple polymer-plasticizer combinations (Hyppölä et al., 1996; Lin et al., 2000;

290 Ljungberg and Wesslén, 2002, 2003; Pillin et al., 2006; Qussi and Suess, 2006). In spite of the 
presence of griseofulvin nanoparticles (15-20 wt $\%$, Section 3.3.3), addition of plasticizer shows a clear impact on the $T_{g}$ of the films. This suggests that poorly water-soluble drug nanoparticles can be incorporated into polymer films without interfering with the ability of plasticizers to interact with the film-forming polymer. PEG-400 led to the greatest depression of $\mathrm{T}_{\mathrm{g}}$ per wt $\%$ 295 plasticizer, followed by glycerin and triacetin.

\subsubsection{Mechanical properties}

The role of plasticizers in influencing the mechanical properties of drug-free polymer films has been thoroughly investigated in literature (Aulton et al., 1981; Bodmeier and

300 Paeratakul, 1994; Entwistle and Rowe, 1979; Gutiérrez-Rocca and McGinity, 1994; Hutchings et al., 1994; Hyppölä et al., 1996; Lim and Hoag, 2013; McHugh and Krochta, 1994). The same has also been investigated for drug-loaded films produced via hot melt extrusion (Repka et al., 1999; Zhu et al., 2006) and solvent casting (Kianfar et al., 2011; Pongjanyakul and Puttipipatkhachorn, 2007). The consensus is that addition of plasticizer to a polymer film formulation leads to a

305 decrease in film strength and an increase in elasticity. However, it is unknown to what extent the influence of plasticizers applies to strip films loaded with poorly water-soluble drug nanoparticles in terms of film mechanical properties.

Fig. 3 depicts the influence of glycerin, triacetin, and PEG-400 on the mechanical properties of HPMC films loaded with griseofulvin nanoparticles. Films with increasing 310 plasticizer content all exhibited decreases in TS, YS, and YM, regardless of which plasticizer was used, suggesting that HPMC films containing poorly water-soluble drug nanoparticles can be effectively plasticized with a variety of plasticizers. Likewise, a general increasing trend in EB was observed with increasing plasticizer content in glycerin and PEG-400 films, although 
there was no such increase in EB for triacetin films, which has been previously reported in

315 ethylcellulose films (Hyppölä et al., 1996). The order of extent of plasticization according to YS and $\mathrm{YM}$ was $\mathrm{PEG}>$ glycerin > triacetin, which was in line with the trend in $\mathrm{T}_{\mathrm{g}}$ depression observed via DSC (Section 3.3.1). This further supports that the presence of poorly water-soluble drug nanoparticles does not interfere with the ability of plasticizers to predictably influence polymer film mechanical properties. The general similarity between trends exhibited in most

320 mechanical properties with increasing plasticizer content for glycerin, triacetin, and PEG-400 implies that all three plasticizers were similarly effective for HPMC films with embedded drug nanoparticles.

\subsubsection{Drug content and uniformity}

Consistency of drug dosage is critical in any pharmaceutical process. In order to assess the drug content of each film sample and ensure even distribution of drug throughout each film, content uniformity tests were performed for all film formulations. As seen in Table 3, all films exhibited $6 \%$ R.S.D. or less in terms of film thickness and griseofulvin mass variation, despite the fact that the size of each sample was roughly one tenth the size of a typical film dosage

330 (while smaller sample sizes were selected to provide better discrimination between formulations in terms of content uniformity, it is expected that equivalent R.S.D. values for $6 \mathrm{~cm}^{2}$ samples would be roughly $1.0-1.5 \%$; results not shown). In addition, triacetin, PEG-400, and plasticizerfree films exhibited $2 \%$ R.S.D. or less in terms of wt $\%$ griseofulvin, while glycerin films had $4 \%$ R.S.D. or less. These results suggest that uniform films with embedded drug nanoparticles can be

335 produced with a variety of plasticizers and plasticizer concentrations within the range studied. Film thickness generally increased with increasing plasticizer content due to the fact that 
addition of plasticizer displaced water from the film precursor formulation (Table 1), resulting in less moisture loss, and consequently less thickness reduction during the drying process. The same reasoning applies to the decreasing trend in wt $\%$ griseofulvin in films with greater 340 plasticizer content, since the residual mass after drying increased with increasing plasticizer content due to less moisture loss.

\subsubsection{Dissolution}

Dissolution curves for all seven fresh films are presented in Fig. 4. By visual inspection

345 of the dissolution curves, it is clear that, with the exception of variation in maximum percentage of griseofulvin released, there is little difference in dissolution rate between the seven film formulations despite their differences in plasticizer content and, consequently, mechanical properties. This is further supported by pairwise comparison of the dissolution curves using similarity and difference factors (Boateng et al., 2012; Costa and Lobo, 2001), according to

350 which the only two films that exhibited statistically significant differences in dissolution rate relative to the other five formulations were $2.5 \%$ triacetin and 2.5\% PEG (all other pairwise curve comparisons were statistically similar; see Supplementary Material). This result demonstrates that the mechanical properties of polymer films loaded with poorly water-soluble drug nanoparticles can be successfully manipulated by multiple plasticizers with no significant 355 impact on the rate of drug release from the film.

While investigation of the impact of various CMAs on dissolution of poorly watersoluble drugs from polymer films is ongoing, previous work suggests that, with adequate dispersion and stabilization of drug nanoparticles within the film, the major controlling factor in determining drug release rate is the polymer matrix. Regarding particle size, Sievens-Figueroa et 
360 al. (2012b) observed faster dissolution rates for griseofulvin nanoparticles as opposed to microparticles from HPMC films under the same dissolution conditions used in this study, while Krull et al. (2016) observed the same from pullulan films in deionized water. Both of these studies reiterate the criticality of preserving poorly water-soluble drug particle size in order to maintain enhanced bioavailability upon delivery. Since griseofulvin particle size was maintained

365 for all seven formulations in this study (Section 3.2), this was not expected to be a source of difference in film dissolution rate. Given proper control of drug particle size, others have demonstrated the impact of other CMAs on poorly water-soluble drug dissolution from films, such as added disintegrants (Susarla et al., 2015) and the drug itself (Krull et al., 2015b), due to their influence on polymer erosion (additional CMAs, including film-forming polymer molecular 370 weight and drug loading, will be the subjects of future work). However, given the hydrophilic and swellable nature of HPMC, the type and amount of plasticizer added did not appear to have a significant influence on the erosion rate of HPMC, resulting in similar dissolution profiles for all films.

While one might have anticipated a direct connection between film mechanical strength 375 and dissolution, this relationship is not a simple one. On the one hand, previous work has shown that films with greater mechanical strength may exhibit slower drug release. Prodduturi et al. (2005) observed slower dissolution for films with greater mechanical strength when varying film-forming polymer molecular weight for hot-melt extruded poly(ethylene oxide) films containing clotrimazole. A similar correlation was found by Krull et al. (2015b) who observed 380 that incorporation of different drug nanoparticles into HPMC films led to films of varying mechanical strength, which coincided with their respective differences in dissolution rate. On the other hand, other work, including this study, has suggested that film mechanical strength does 
not necessarily affect drug release. Pongjanyakul and Puttipipatkhachorn (2007) observed similar drug permeation rates between acetaminophen tablets coated by sodium-alginate-magnesium

385 aluminum silicate films with varying amounts of glycerin and PEG-400, despite significant differences in the mechanical properties of the film coatings. Lin et al. (1995) noted little difference in permeation profiles of piroxicam from Eudragit E films with varying mechanical properties due to incorporation of four different plasticizers. Krull et al. (2016) observed similar dissolution rates from GF nanoparticle-laden films of various drug loadings despite significant

390 differences in their mechanical properties. Nonetheless, plasticizer has been shown to be a unique tuning parameter for film mechanical properties that does not appear to impact drug release, unlike film-forming polymer properties, such as molecular weight, which have been shown to affect both. These results suggest that the relationship between film mechanical properties and dissolution is more complex than it first appears, and further investigation is 395 required to elucidate a possible connection.

\subsubsection{Thermogravimetric analysis (TGA)}

TGA was performed on all film formulations and normalized to account for slight variations in griseofulvin and SDS content between films according to Susarla et al. (2013) (Fig.

400 5). All films exhibited a weight loss between $1.5 \%$ and $4.5 \%$ up to $100{ }^{\circ} \mathrm{C}$, which can be attributed to the loss of water within the film. 15 min exposure to $150{ }^{\circ} \mathrm{C}$ resulted in varying degrees of wt\% drops between films depending on which and how much plasticizer each film contained, suggesting that this drop, or lack thereof, was due to the presence or absence of plasticizer in the film formulation. Consequently, the plasticizer-free film exhibited a negligible 405 drop in wt\% during this time. The extent of weight loss up to $250{ }^{\circ} \mathrm{C}$ between the three 
plasticizers was glycerin > triacetin > PEG-400, and films with higher plasticizer concentration experienced greater drops in wt $\%$ due to the loss of additional plasticizer.

\subsubsection{Scanning electron microscopy (SEM)}

Cross-sectional SEM images of all seven film formulations were taken in order to qualitatively assess the size and morphology of the embedded API particles (Fig. 6). As can be clearly seen in all seven images, the griseofulvin nanoparticles were finely dispersed throughout the films with no visible signs of aggregation. This reinforces the assessment of the redispersion results in Section 2.3.2 that the polymer matrix is able to physically stabilize the embedded 415 griseofulvin nanoparticles regardless of the plasticizer or plasticizer content under investigation.

\subsubsection{Long-term stability of films}

In order to evaluate the ability of the film format to preserve drug nanoparticle size and enhanced dissolution over an extended period of time, redispersion and dissolution tests were 420 performed for all formulations over the course of 6 months' storage at $40{ }^{\circ} \mathrm{C}$ and $75 \% \mathrm{RH}$. First, the long-term physical stability of the griseofulvin nanoparticles embedded within the polymer matrix was assessed via redispersion (Table 2). While there was some variation in the $d_{10}, d_{50}$, and $d_{90}$ values of redispersed griseofulvin particles between formulations, little variation was observed within any of the seven film formulations during this time. This suggests that long-term

425 stability of poorly water-soluble drug nanoparticles is indeed achievable in polymer strip films containing various plasticizers and plasticizer content.

In order to investigate the impact of long-term storage on the dissolution rate of the films, Fig. 7 shows dissolution curves for all seven film formulations after 0,3 , and 6 months of storage 
at $40{ }^{\circ} \mathrm{C}$ and $75 \% \mathrm{RH}$. While some films exhibited variation in maximum percentage of

430 griseofulvin released before and after storage, little statistical difference in dissolution rate was observed for most of the seven film formulations (see Supplementary Material). This suggests that polymer films are capable of successfully stabilizing and releasing poorly water-soluble drug nanoparticles at a consistent rate, even after long-term storage.

\section{4. Conclusions}

The objective of this work was to investigate the impact of plasticizer and plasticizer concentration on various properties of strip films loaded with poorly water-soluble drug (griseofulvin) nanoparticles. Addition of any of the three plasticizers under investigation (glycerin, triacetin, and PEG-400) led to a depression of film glass transition temperature, a

440 decrease in film tensile strength, and an increase in film elongation at break. However, in spite of these clear differences, there was little difference between the dissolution rates of the films, perhaps due to the robustness with which the drug nanoparticles were stabilized and dispersed. This suggests that film mechanical properties may be successfully manipulated by adjusting the amount of plasticizer used without impacting the rate of drug release from the films. The

445 similarity between dissolution rates of different films was observed even after the films were stored under stress conditions for six months, demonstrating that the consistency of drug release between formulations is preserved after long-term storage. In addition, the redispersibility of the embedded griseofulvin nanoparticles was also preserved after six months' storage, demonstrating the long-term stability of the films. This consistency between films with different plasticizers and 450 plasticizer concentrations will allow formulators the freedom to adjust the strength and elasticity 
of films containing poorly water-soluble drug without negatively impacting drug release or other film properties.

\section{Conflict of interest}

The authors declare that there are no conflicts of interest.

\section{Acknowledgements}

The authors are grateful for financial support from the National Science Foundation (NSF) in part through the ERC (EEC-0540855) award and from the National Institute of Health

(NIH) NIH-U01 in part through award U01FD005521. We also thank Dr. R. Susarla for her valuable input on experimental design and Ms. S. Legarda for her assistance with preliminary studies.

\section{References}

465 Aulton, M.E., Abdul-Razzak, M.H., Hogan, J.E., 1981. The mechanical properties of hydroxypropylmethylcellulose films derived from aqueous systems Part 1: The influence of plasticisers. Drug Dev. Ind. Pharm. 7, 649-668.

Beck, C., Sievens-Figueroa, L., Gärtner, K., Jerez-Rozo, J.I., Romañach, R.J., Bilgili, E., Davé, R.N., 2013. Effects of stabilizers on particle redispersion and dissolution from polymer strip films containing liquid antisolvent precipitated griseofulvin particles. Powder Technol. 236, 37-51.

Bhakay, A., Azad, M., Vizzotti, E., Dave, R.N., Bilgili, E., 2014. Enhanced recovery and dissolution of griseofulvin nanoparticles from surfactant-free nanocomposite microparticles incorporating wet-milled swellable dispersants. Drug Dev. Ind. Pharm. 40, $1509-1522$.

Bhakay, A., Davé, R., Bilgili, E., 2013. Recovery of BCS Class II drugs during aqueous redispersion of core-shell type nanocomposite particles produced via fluidized bed coating. Powder Technol. 236, 221-234.

Bhakay, A., Vizzotti, E., Li, M., Davé, R., Bilgili, E., 2016. Incorporation of fenofibrate nanoparticles prepared by melt emulsification into polymeric films. J. Pharm. Innov. 11, 53-63. 
Bilgili, E., Afolabi, A., 2012. A combined microhydrodynamics-polymer adsorption analysis for elucidation of the roles of stabilizers in wet stirred media milling. Int. J. Pharm. 439, 193206.

485 Boateng, J.S., Matthews, K.H., Auffret, A.D., Humphrey, M.J., Eccleston, G.M., Stevens, H.N., 2012. Comparison of the in vitro release characteristics of mucosal freeze-dried wafers and solvent-cast films containing an insoluble drug. Drug Dev. Ind. Pharm. 38, 47-54.

Bodmeier, R., Paeratakul, O., 1994. Mechanical properties of dry and wet cellulosic and acrylic films prepared from aqueous colloidal polymer dispersions used in the coating of solid 490 dosage forms. Pharm. Res. 11, 882-888.

Borges, A.F., Silva, C., Coelho, J.F.J., Simões, S., 2015a. Oral films: Current status and future perspectives II - Intellectual property, technologies and market needs. J. Controlled Release 206, 108-121.

Borges, A.F., Silva, C., Coelho, J.F.J., Simões, S., 2015b. Oral films: Current status and future perspectives: I - Galenical development and quality attributes. J. Controlled Release 206, 1-19.

Bruce, L.D., McGinity, J.W., 2008. Polymer interactions with drugs and excipients, in: McGinity, J.W., Felton, L.A. (Eds.), Aqueous Polymeric Coatings for Pharmaceutical Dosage Forms, third ed. CRC Press, New York, pp. 369-408.

500 Costa, P., Lobo, J.M.S., 2001. Modeling and comparison of dissolution profiles. Eur. J. Pharm. Sci. 13, 123-133.

Dave, R.H., Shah, D.A., Patel, P.G., 2014. Development and evaluation of high loading oral dissolving film of aspirin and acetaminophen. J. Pharm. Sci. Pharmacol. 1, 112-122.

Davé, R.N., Susarla, R., Bilgili, E., Sievens-Figueroa, L., Khusid, B., Muzzio, F., Bhakay, A., 2014. System and method for fabrication of uniform polymer films containing nano and micro particles via continuous drying process. PCT Application No. PCT/US14/30506. Filed March 17, 2014.

de Villiers, M.M., 1996. Influence of agglomeration of cohesive particles on the dissolution behaviour of furosemide powder. Int. J. Pharm. 136, 175-179.

510 Dixit, R.P., Puthli, S.P., 2009. Oral strip technology: Overview and future potential. J. Controlled Release 139, 94-107.

Entwistle, C.A., Rowe, R.C., 1979. Plasticization of cellulose ethers used in the film coating of tablets. J. Pharm. Pharmacol. 31, 269-272.

515 preparation of fast-dissolving oral films. J. Pharm. Pharmacol. 62, 539-545.

Gómez-Carracedo, A., Alvarez-Lorenzo, C., Gómez-Amoza, J.L., Concheiro, A., 2003. Chemical structure and glass transition temperature of non-ionic cellulose ethers. J. Therm. Anal. Calorim. 73, 587-596.

Gottnek, M., Süvegh, K., Pintye-Hódi, K., Regdon Jr, G., 2013. Effects of excipients on the tensile strength, surface properties and free volume of Klucel® free films of pharmaceutical importance. Radiat. Phys. Chem. 89, 57-63.

Gutiérrez-Rocca, J.C., McGinity, J.W., 1994. Influence of water soluble and insoluble plasticizers on the physical and mechanical properties of acrylic resin copolymers. Int. J. Pharm. 103, 293-301.

525 Hoffmann, E.M., Breitenbach, A., Breitkreutz, J., 2011. Advances in orodispersible films for drug delivery. Expert Opin. Drug Deliv. 8, 299-316. 
Honary, S., Orafai, H., 2002. The effect of different plasticizer molecular weights and concentrations on mechanical and thermomechanical properties of free films. Drug Dev. Ind. Pharm. 28, 711-715.

530 Hutchings, D., Clarson, S., Sakr, A., 1994. Studies of the mechanical properties of free films prepared using an ethylcellulose pseudolatex coating system. Int. J. Pharm. 104, 203-213.

Hyppölä, R., Husson, I., Sundholm, F., 1996. Evaluation of physical properties of plasticized ethyl cellulose films cast from ethanol solution Part I. Int. J. Pharm. 133, 161-170.

Kararli, T.T., Hurlbut, J.B., Needham, T.E., 1990. Glass-rubber transitions of cellulosic polymers by dynamic mechanical analysis. J. Pharm. Sci. 79, 845-848.

Kesisoglou, F., Panmai, S., Wu, Y., 2007. Nanosizing - Oral formulation development and biopharmaceutical evaluation. Adv. Drug Deliv. Rev. 59, 631-644.

Kianfar, F., Chowdhry, B.Z., Antonijevic, M.D., Boateng, J.S., 2011. Novel films for drug delivery via the buccal mucosa using model soluble and insoluble drugs. Drug Dev. Ind.

540 Pharm. 38, 1207-1220.

Kipp, J.E., 2004. The role of solid nanoparticle technology in the parenteral delivery of poorly water-soluble drugs. Int. J. Pharm. 284, 109-122.

Krull, S.M., Li, M., Bilgili, E., Davé, R.N., 2015a. Polymer strip films for delivery of poorly water-soluble drugs. Am. Pharm. Rev. 18, 48-52.

545 Krull, S.M., Ma, Z., Li, M., Davé, R.N., Bilgili, E., 2016. Preparation and characterization of fast dissolving pullulan films containing BCS class II drug nanoparticles for bioavailability enhancement. Drug Dev. Ind. Pharm. 42, 1073-1085.

Krull, S.M., Susarla, R., Afolabi, A., Li, M., Ying, Y., Iqbal, Z., Bilgili, E., Davé, R.N., $2015 b$. Polymer strip films as a robust, surfactant-free platform for delivery of BCS Class II drug

$550 \quad$ nanoparticles. Int. J. Pharm. 489, 45-57.

Kumar, G.P., Phani, A.R., Prasad, R.G.S.V., Sanganal, J.S., Manali, N., Gupta, R., Rashmi, N., Prabhakara, G.S., Salins, C.P., Sandeep, K., Raju, D.B., 2014. Polyvinylpyrrolidone oral films of enrofloxacin: Film characterization and drug release. Int. J. Pharm. 471, 146-152.

Lai, F., Franceschini, I., Corrias, F., Sala, M.C., Cilurzo, F., Sinico, C., Pini, E., 2015. Maltodextrin fast dissolving films for quercetin nanocrystal delivery. A feasibility study. Carbohydr. Polym. 121, 217-223.

Lim, H., Hoag, S., 2013. Plasticizer effects on physical-mechanical properties of solvent cast Soluplus ${ }^{\circledR}$ films. AAPS PharmSciTech 14, 903-910.

Lin, S.-Y., Chen, K.-S., Run-Chu, L., 2000. Organic esters of plasticizers affecting the water absorption, adhesive property, glass transition temperature and plasticizer permanence of Eudragit acrylic films. J. Controlled Release 68, 343-350.

Lin, S.Y., Lee, C.J., Lin, Y.Y., 1995. Drug-polymer interaction affecting the mechanical properties, adhesion strength and release kinetics of piroxicam-loaded Eudragit E films plasticized with different plasticizers. J. Controlled Release 33, 375-381.

565 Ljungberg, N., Wesslén, B., 2002. The effects of plasticizers on the dynamic mechanical and thermal properties of poly(lactic acid). J. Appl. Polym. Sci. 86, 1227-1234.

Ljungberg, N., Wesslén, B., 2003. Tributyl citrate oligomers as plasticizers for poly (lactic acid): thermo-mechanical film properties and aging. Polymer 44, 7679-7688.

$570 \quad$ humidity and plasticizer concentration on the water content and glass transition of starch materials. Polymer 38, 5401-5406. 
McHugh, T.H., Krochta, J.M., 1994. Sorbitol- vs glycerol-plasticized whey protein edible films: Integrated oxygen permeability and tensile property evaluation. J. Agric. Food Chem. 42, 841-845.

575 McPhillips, H., Craig, D.Q.M., Royall, P.G., Hill, V.L., 1999. Characterisation of the glass transition of HPMC using modulated temperature differential scanning calorimetry. Int. J. Pharm. 180, 83-90.

Monteiro, A., Afolabi, A., Bilgili, E., 2013. Continuous production of drug nanoparticle suspensions via wet stirred media milling: A fresh look at the Rehbinder effect. Drug Dev. Ind. Pharm. 39, 266-283.

Murdande, S.B., Shah, D.A., Dave, R.H., 2015. Impact of nanosizing on solubility and dissolution rate of poorly soluble pharmaceuticals. J. Pharm. Sci. 104, 2094-2102.

Nyamweya, N., Hoag, S., 2000. Assessment of Polymer-Polymer Interactions in Blends of HPMC and Film Forming Polymers by Modulated Temperature Differential Scanning Calorimetry. Pharm. Res. 17, 625-631.

Panda, B., Parihar, A.S., Mallick, S., 2014. Effect of plasticizer on drug crystallinity of hydroxypropyl methylcellulose matrix film. Int. J. Biol. Macromol. 67, 295-302.

Pillin, I., Montrelay, N., Grohens, Y., 2006. Thermo-mechanical characterization of plasticized PLA: Is the miscibility the only significant factor? Polymer 47, 4676-4682.

590 Pongjanyakul, T., Puttipipatkhachorn, S., 2007. Alginate-magnesium aluminum silicate films: Effect of plasticizers on film properties, drug permeation and drug release from coated tablets. Int. J. Pharm. 333, 34-44.

Prodduturi, S., Manek, R.V., Kolling, W.M., Stodghill, S.P., Repka, M.A., 2005. Solid-state stability and characterization of hot-melt extruded poly (ethylene oxide) films. J. Pharm.

595 Sci. 94, 2232-2245.

Qussi, B., Suess, W.G., 2006. The influence of different plasticizers and polymers on the mechanical and thermal properties, porosity and drug permeability of free shellac films. Drug Dev. Ind. Pharm. 32, 403-412.

600

Repka, M.A., Gerding, T.G., Repka, S.L., McGinity, J.W., 1999. Influence of plasticizers and drugs on the physical-mechanical properties of hydroxypropylcellulose films prepared by hot melt extrusion. Drug Dev. Ind. Pharm. 25, 625-633.

Sakellariou, P., Rowe, R.C., White, E.F.T., 1986. An evaluation of the interaction and plasticizing efficiency of the polyethylene glycols in ethyl cellulose and hydroxypropyl methylcellulose films using the torsional braid pendulum. Int. J. Pharm. 31, 55-64.

605 Shen, B.d., Shen, C.y., Yuan, X.d., Bai, J.x., Lv, Q.y., Xu, H., Dai, L., Yu, C., Han, J., Yuan, H.l., 2013. Development and characterization of an orodispersible film containing drug nanoparticles. Eur. J. Pharm. Biopharm. 85, 1348-1356.

Sievens-Figueroa, L., Bhakay, A., Jerez-Rozo, J.I., Pandya, N., Romañach, R.J., Michniak-Kohn, B., Iqbal, Z., Bilgili, E., Davé, R.N., 2012a. Preparation and characterization of hydroxypropyl methyl cellulose films containing stable BCS Class II drug nanoparticles for pharmaceutical applications. Int. J. Pharm. 423, 496-508.

Sievens-Figueroa, L., Pandya, N., Bhakay, A., Keyvan, G., Michniak-Kohn, B., Bilgili, E., Davé, R., 2012b. Using USP I and USP IV for discriminating dissolution rates of nano- and microparticle-loaded pharmaceutical strip-films. AAPS PharmSciTech 13, 1473-1482.

615 Susarla, R., Afolabi, A., Patel, D., Bilgili, E., Davé, R.N., 2015. Novel use of superdisintegrants as viscosity enhancing agents in biocompatible polymer films containing griseofulvin nanoparticles. Powder Technol. 285, 25-33. 
Susarla, R., Sievens-Figueroa, L., Bhakay, A., Shen, Y., Jerez-Rozo, J.I., Engen, W., Khusid, B., Bilgili, E., Romañach, R.J., Morris, K.R., Michniak-Kohn, B., Davé, R.N., 2013. Fast drying of biocompatible polymer films loaded with poorly water-soluble drug nanoparticles via low temperature forced convection. Int. J. Pharm. 455, 93-103.

Thakhiew, W., Devahastin, S., Soponronnarit, S., 2010. Effects of drying methods and plasticizer concentration on some physical and mechanical properties of edible chitosan films. J. Food Eng. 99, 216-224.

625 Visser, J.C., Woerdenbag, H.J., Crediet, S., Gerrits, E., Lesschen, M.A., Hinrichs, W.L.J., Breitkreutz, J., Frijlink, H.W., 2015. Orodispersible films in individualized pharmacotherapy: The development of a formulation for pharmacy preparations. Int. J. Pharm. 478, 155-163.

Wypych, G., 2004. Handbook of plasticizers. ChemTec Publishing, New York.

630 Zhu, Y., Mehta, K.A., McGinity, J.W., 2006. Influence of plasticizer level on the drug release from sustained release film coated and hot-melt extruded dosage forms. Pharm. Dev. Technol. 11, 285-294. 


\section{List of figures}

Fig. 1. Low shear $\left(2.2 \mathrm{~s}^{-1}\right)$ room temperature viscosity of polymer solutions and film precursor suspensions. Values are mean \pm S.D., $\mathrm{n}=7$.

Fig. 2. Glass transition temperature of griseofulvin nanoparticle-laden HPMC films containing various amounts of plasticizer.

Fig. 3. (a) Tensile strength, (b) yield strength, (c) Young's modulus, and (d) percent elongation at break of HPMC films with different plasticizers and content containing griseofulvin nanoparticles. Values are mean \pm S.D., $n=4$.

Fig. 4. Comparison of dissolution profiles between HPMC films loaded with griseofulvin nanoparticles containing different plasticizers and plasticizer content. Values are mean \pm S.D., $n$ $=6$.

Fig. 5. Normalized TGA curves for HPMC films with different plasticizers and plasticizer content loaded with griseofulvin nanoparticles.

Fig. 6. Cross sectional SEM images of HPMC films loaded with griseofulvin nanoparticles made from polymer solutions containing (a) no plasticizer, (b) $2.5 \%$ glycerin, (c) $5.0 \%$ glycerin, (d) 2.5\% triacetin, (e) $5.0 \%$ triacetin, (f) $2.5 \%$ PEG, and (g) 5.0\% PEG. Scale bars are $1 \mu \mathrm{m}$ for (a-f) and $2 \mu \mathrm{m}$ for $(\mathrm{g})$.

Fig. 7. Comparison of dissolution profiles for all films immediately after film preparation, after 3 months of storage at $40{ }^{\circ} \mathrm{C}, 75 \% \mathrm{RH}$, and after 6 months of storage at $40{ }^{\circ} \mathrm{C}, 75 \% \mathrm{RH}$. Values are mean \pm S.D., $n=6$. 


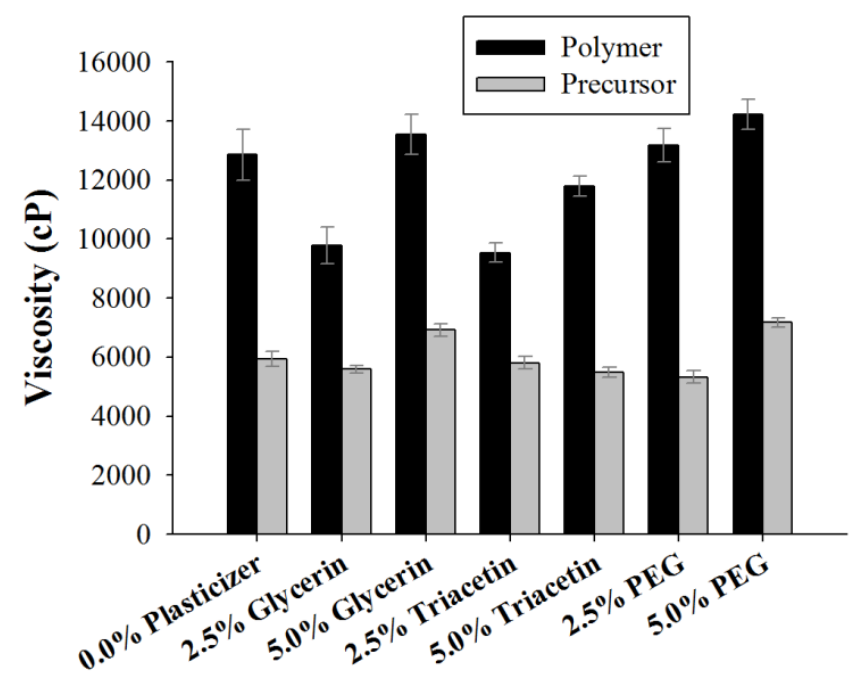

Fig. 1. Krull et al. (2016) intended for Eur J Pharm Sci.

Low shear $\left(2.2 \mathrm{~s}^{-1}\right)$ room temperature viscosity of polymer solutions and film precursor suspensions. Values are mean \pm S.D., $\mathrm{n}=7$. 


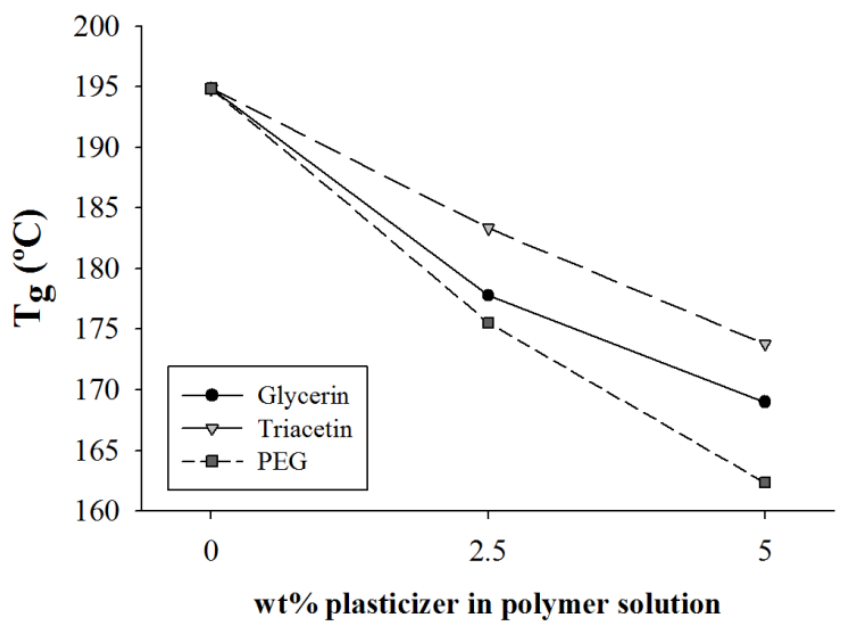

Fig. 2. Krull et al. (2016) intended for Eur J Pharm Sci.

Glass transition temperature of griseofulvin nanoparticle-laden HPMC films containing various amounts of plasticizer. 


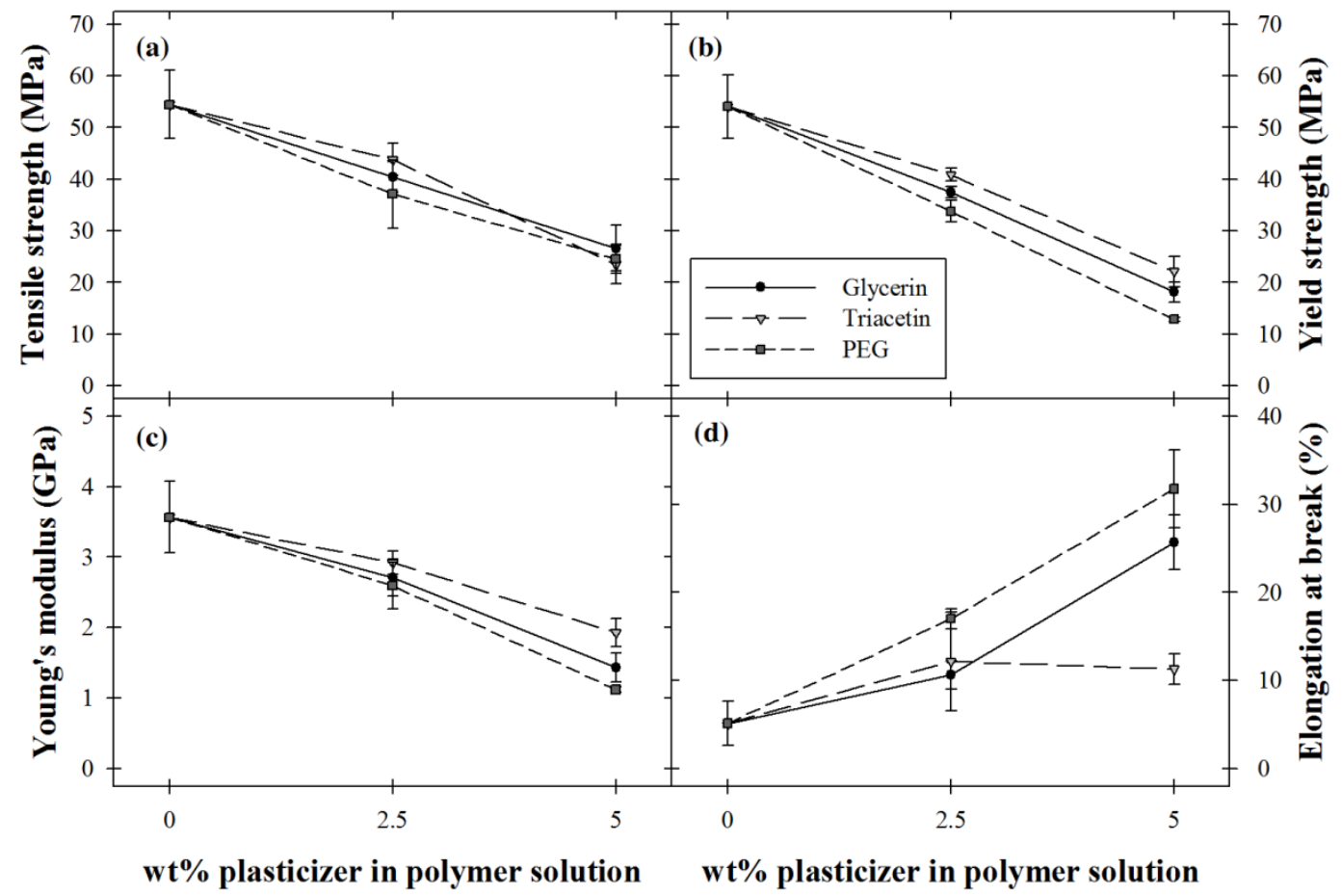

Fig. 3. Krull et al. (2016) intended for Eur J Pharm Sci.

(a) Tensile strength, (b) yield strength, (c) Young's modulus, and (d) percent elongation at break of HPMC films with different plasticizers and content containing griseofulvin nanoparticles. Values are mean \pm S.D., $n=4$. 


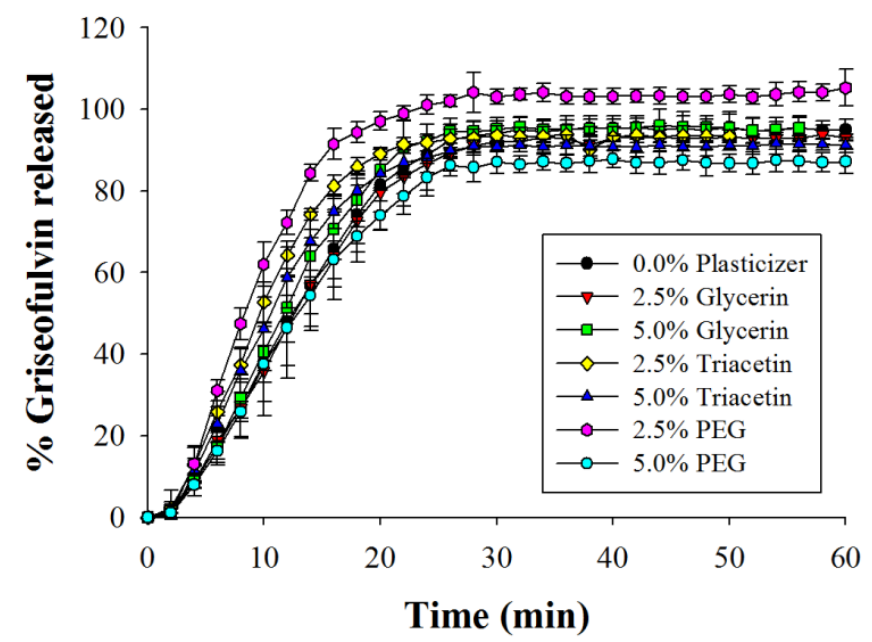

Fig. 4. Krull et al. (2016) intended for Eur J Pharm Sci.

Comparison of dissolution profiles between HPMC films loaded with griseofulvin nanoparticles containing different plasticizers and plasticizer content. Values are mean \pm S.D., $\mathrm{n}=6$. 


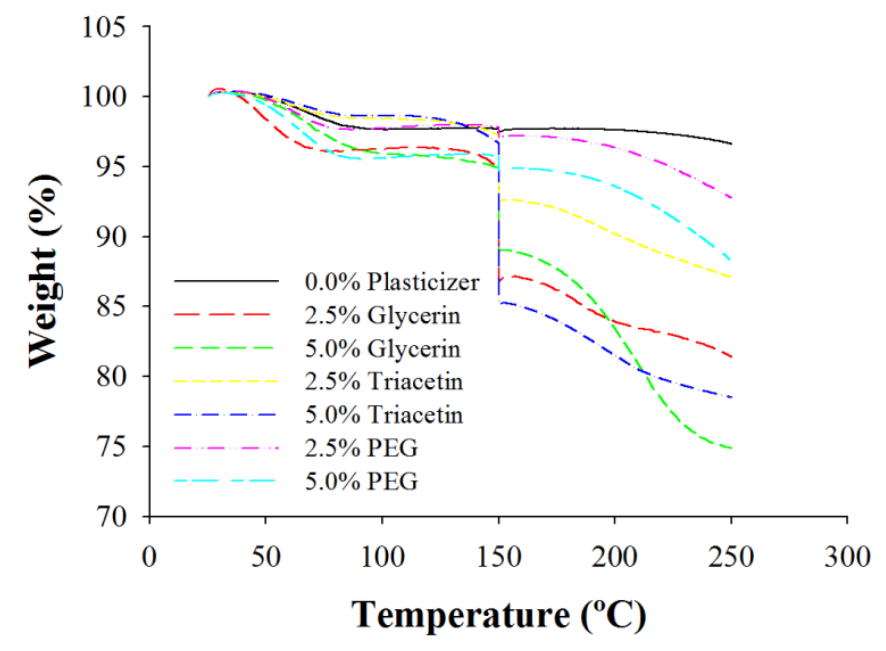

Fig. 5. Krull et al. (2016) intended for Eur J Pharm Sci.

Normalized TGA curves for HPMC films with different plasticizers and plasticizer content loaded with griseofulvin nanoparticles. 

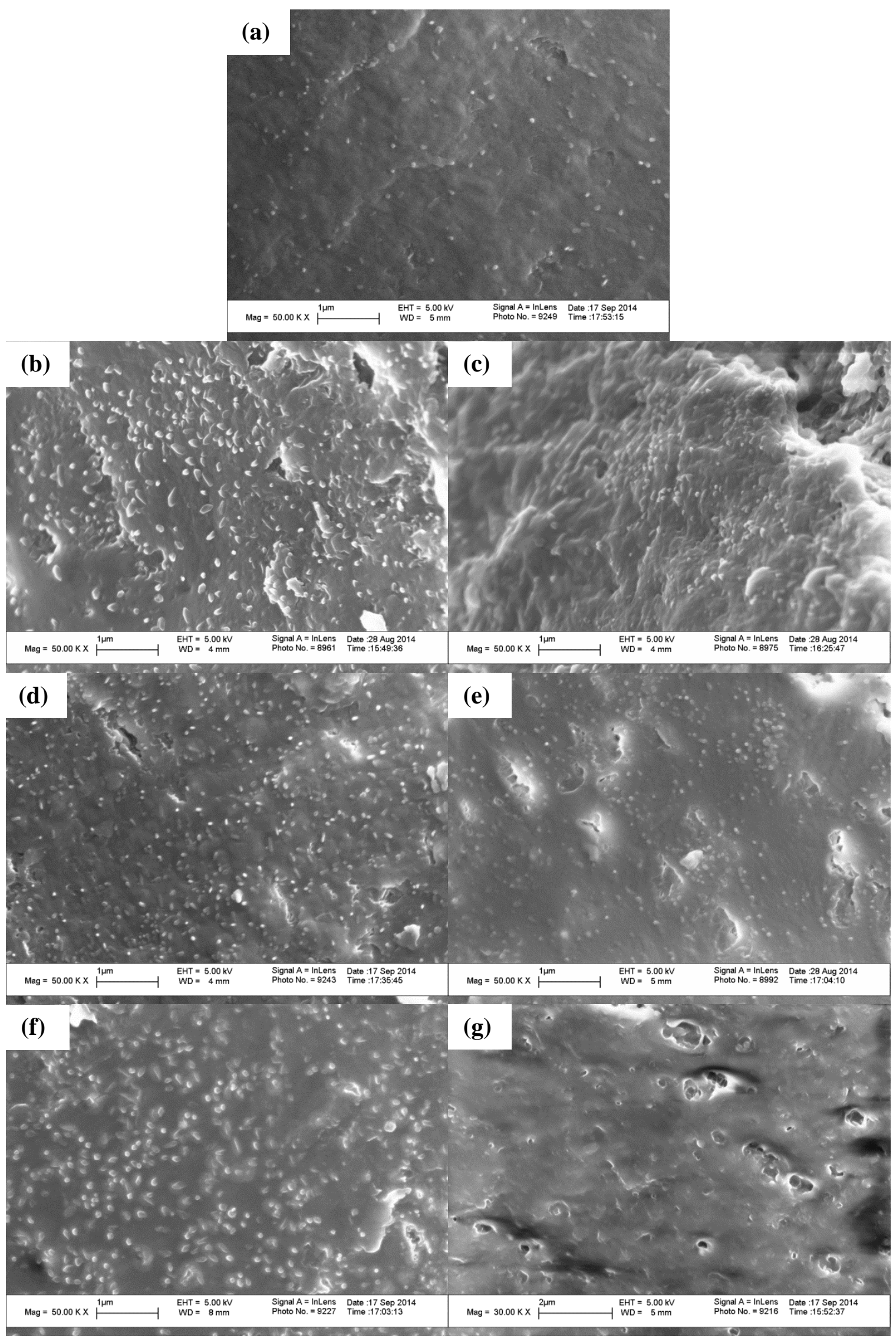
Fig. 6. Krull et al. (2016) intended for Eur J Pharm Sci.

Cross sectional SEM images of HPMC films loaded with griseofulvin nanoparticles made from polymer solutions containing (a) no plasticizer, (b) $2.5 \%$ glycerin, (c) $5.0 \%$ glycerin, (d) $2.5 \%$ triacetin, (e) 5.0\% triacetin, (f) 2.5\% PEG, and (g) 5.0\% PEG. Scale bars are $1 \mu \mathrm{m}$ for (a-f) and $2 \mu \mathrm{m}$ for $(\mathrm{g})$. 


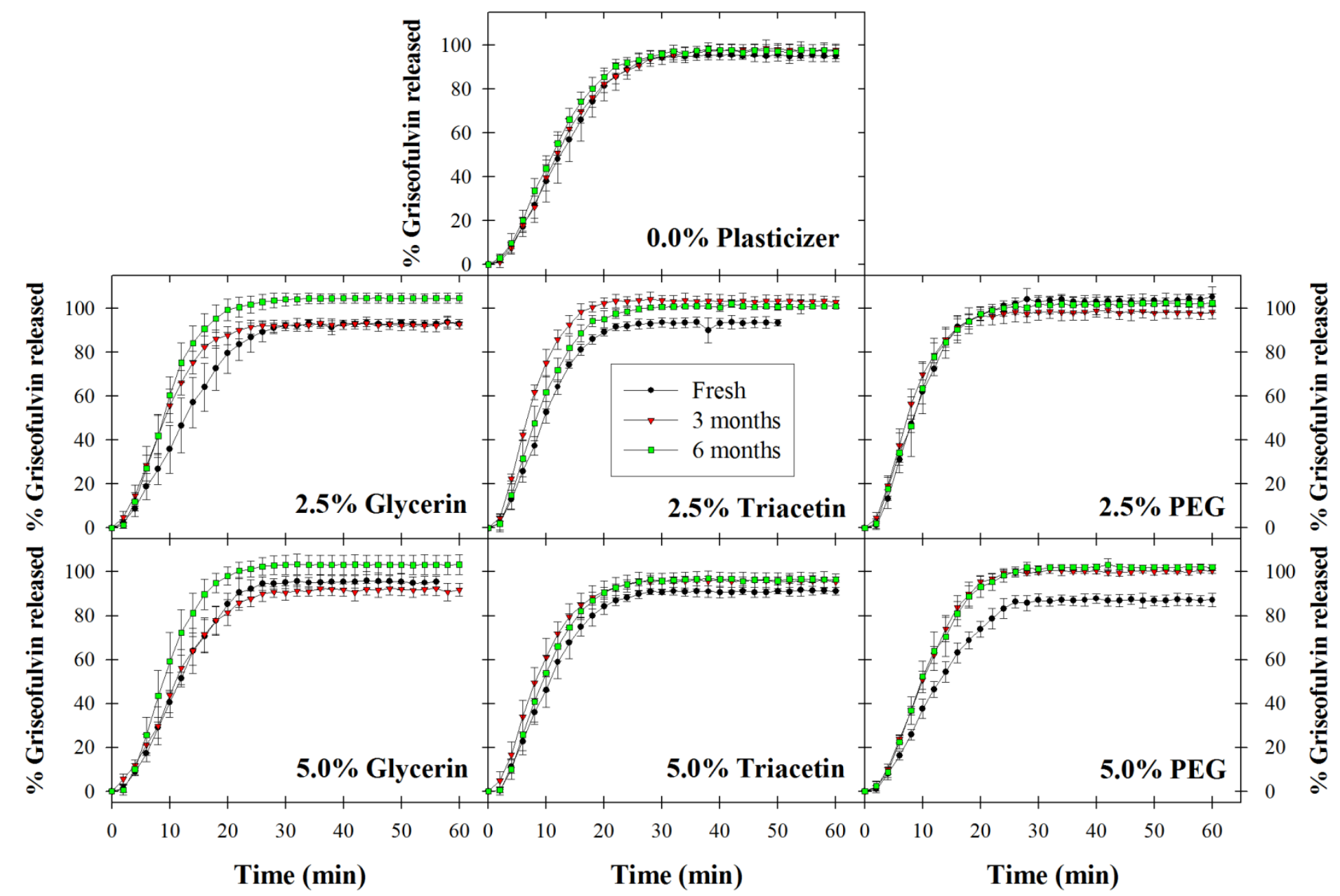

Fig. 7. Krull et al. (2016) intended for Eur J Pharm Sci.

Comparison of dissolution profiles for all films immediately after film preparation, after 3 months of storage at $40{ }^{\circ} \mathrm{C}, 75 \% \mathrm{RH}$, and after 6 months of storage at $40{ }^{\circ} \mathrm{C}, 75 \%$ RH. Values are mean \pm S.D., $n=6$. 


\section{List of tables}

Table 1. Film-forming polymer solution formulations prior to mixing with griseofulvin nanosuspension.

Table 2. Particle size distributions of fresh griseofulvin nanosuspension and redispersed film samples immediately after film preparation, after 3 months of storage at $40{ }^{\circ} \mathrm{C}, 75 \% \mathrm{RH}$, and after 6 months of storage at $40{ }^{\circ} \mathrm{C}, 75 \% \mathrm{RH}$.

Table 3. Content uniformity of HPMC films with different plasticizers and content. Values are an average of 10 samples $\sim 0.7 \mathrm{~cm}^{2}$ in area. 


\section{Table 1}

Film-forming polymer solution formulations prior to mixing with griseofulvin nanosuspension.

\begin{tabular}{ccccc}
\hline Formulation & Plasticizer & wt $\%$ HPMC & wt $\%$ plasticizer & wt\% water \\
\hline $0.0 \%$ Plasticizer & N/A & 15.0 & 0.0 & 85.0 \\
$2.5 \%$ Glycerin & Glycerin & 15.0 & 2.5 & 82.5 \\
$5.0 \%$ Glycerin & Glycerin & 15.0 & 5.0 & 80.0 \\
2.5\% Triacetin & Triacetin & 15.0 & 2.5 & 82.5 \\
$5.0 \%$ Triacetin & Triacetin & 15.0 & 5.0 & 80.0 \\
2.5\% PEG & PEG & 15.0 & 2.5 & 82.5 \\
$5.0 \%$ PEG & PEG & 15.0 & 5.0 & 80.0 \\
\hline
\end{tabular}




\section{Table 2}

Particle size distributions of fresh griseofulvin nanosuspension and redispersed film samples immediately after film preparation, after 3 months of storage at $40{ }^{\circ} \mathrm{C}, 75 \% \mathrm{RH}$, and after 6 months of storage at $40{ }^{\circ} \mathrm{C}, 75 \% \mathrm{RH}$.

\begin{tabular}{|c|c|c|c|c|c|c|c|c|c|}
\hline \multirow[b]{2}{*}{ Formulation } & \multirow[b]{2}{*}{ Fresh } & \multirow{2}{*}{$\begin{array}{l}d_{10}(\mu \mathrm{m}) \\
3 \text { months }\end{array}$} & \multirow[b]{2}{*}{6 months } & \multirow[b]{2}{*}{ Fresh } & \multirow{2}{*}{$\begin{array}{l}d_{50}(\mu \mathrm{m}) \\
3 \text { months }\end{array}$} & \multirow[b]{2}{*}{6 months } & \multirow[b]{2}{*}{ Fresh } & \multirow{2}{*}{$\begin{array}{l}d_{90}(\mu \mathrm{m}) \\
3 \text { months }\end{array}$} & \multirow[b]{2}{*}{6 month } \\
\hline & & & & & & & & & \\
\hline Suspension & 0.099 & -- & -- & 0.159 & -- & -- & 0.262 & -- & -- \\
\hline $0.0 \%$ Plasticizer & 0.104 & 0.095 & 0.115 & 0.231 & 0.172 & 0.179 & 0.509 & 0.309 & 0.296 \\
\hline $5.0 \%$ Glycerin & 0.092 & 0.091 & 0.113 & 0.166 & 0.166 & 0.190 & 0.305 & 0.306 & 0.328 \\
\hline $2.5 \%$ Triacetin & 0.106 & 0.100 & 0.114 & 0.241 & 0.228 & 0.237 & 0.422 & 0.404 & 0.410 \\
\hline $5.0 \%$ PEG & 0.093 & 0.094 & 0.061 & 0.171 & 0.212 & 0.169 & 0.318 & 0.390 & 0.364 \\
\hline
\end{tabular}


Table 3

Content uniformity of HPMC films with different plasticizers and content. Values are an average of 10 samples $\sim 0.7 \mathrm{~cm}^{2}$ in area.

\begin{tabular}{ccccccc}
\hline Formulation & $\begin{array}{c}\text { Thickness } \\
(\mu \mathrm{m})\end{array}$ & $\begin{array}{c}\text { R.S.D. } \\
(\%)\end{array}$ & $\begin{array}{c}\text { Griseofulvin mass per } \\
\text { unit area }\left(\mathrm{mg} / \mathrm{cm}^{2}\right)\end{array}$ & $\begin{array}{c}\text { R.S.D. } \\
(\%)\end{array}$ & $\begin{array}{c}\text { wt\% } \\
\text { griseofulvin }\end{array}$ & $\begin{array}{c}\text { R.S.D. } \\
(\%)\end{array}$ \\
\hline $0.0 \%$ Plasticizer & 86.9 & $3.9 \%$ & 2.48 & $4.2 \%$ & 20.9 & $2.3 \%$ \\
2.5\% Glycerin & 97.1 & $4.8 \%$ & 2.57 & $5.8 \%$ & 18.2 & $3.5 \%$ \\
$5.0 \%$ Glycerin & 108.0 & $2.9 \%$ & 2.16 & $5.5 \%$ & 15.3 & $4.2 \%$ \\
2.5\% Triacetin & 84.4 & $4.4 \%$ & 2.05 & $5.4 \%$ & 18.7 & $1.0 \%$ \\
$5.0 \%$ Triacetin & 86.6 & $6.5 \%$ & 1.99 & $5.9 \%$ & 16.4 & $2.3 \%$ \\
2.5\% PEG & 72.2 & $2.5 \%$ & 1.68 & $1.9 \%$ & 16.6 & $2.2 \%$ \\
$5.0 \%$ PEG & 91.1 & $3.4 \%$ & 1.99 & $3.2 \%$ & 15.4 & $2.2 \%$ \\
\hline
\end{tabular}

